Published in final edited form as:

Addict Behav. 2013 April ; 38(4): 2052-2059. doi:10.1016/j.addbeh.2012.12.023.

\title{
Changes in Alcohol-Related Brain Networks Across the First Year of College: A Prospective Pilot Study Using fMRI Effective Connectivity Mapping
}

\author{
Adriene M. Beltz, \\ Department of Psychology, The Pennsylvania State University \\ Kathleen M. Gates, \\ Department of Human Development and Family Studies and Social, Life, and Engineering \\ Sciences Imaging Center, The Pennsylvania State University
}

Anna S. Engels,

Department of Psychology and Social, Life, and Engineering Sciences Imaging Center, The Pennsylvania State University

Peter C. M. Molenaar,

Departments of Human Development and Family Studies and Psychology, The Pennsylvania State University

Carmen Pulido,

Department of Psychiatry, University of California San Diego

Robert Turrisi,

Department of Biobehavioral Health, The Pennsylvania State University

Sheri A. Berenbaum, Departments of Psychology and Pediatrics, The Pennsylvania State University

Rick O. Gilmore, and Department of Psychology and Social, Life, and Engineering Sciences Imaging Center, The Pennsylvania State University

Stephen J. Wilson

Department of Psychology, The Pennsylvania State University

\begin{abstract}
(c) 2012 Elsevier Ltd. All rights reserved.

Correspondence concerning this article should be addressed to Adriene M. Beltz, Department of Psychology, 382 Moore Building, The Pennsylvania State University, University Park, PA 16802; axb1017@psu.edu.

Contributor

A. Engels, C. Pulido, R. Turrisi, S. Berenbaum, R. Gilmore, and S. Wilson designed the study. A. Engels, R. Turrisi, and R. Gilmore collected the data. A. Engels, C. Pulido, and R. Turrisi contributed research tools. K. Gates and P. Molennar contributed data analytic tools. A. Beltz, K. Gates, A. Engels, P. Molenaar, and R. Gilmore analyzed the data. A. Beltz, K. Gates, A. Engels, P. Molenaar, S. Berenbaum, R. Gilmore, and S. Wilson interpreted the data. A. Beltz, S. Berenbaum, R. Gilmore, and S. Wilson conceptualized the writing of the manuscript. A. Beltz, K. Gates, A. Engels, and S. Wilson wrote the manuscript.
\end{abstract}

Conflict of Interest

Authors declare that they have no conflicts of interest.

Publisher's Disclaimer: This is a PDF file of an unedited manuscript that has been accepted for publication. As a service to our customers we are providing this early version of the manuscript. The manuscript will undergo copyediting, typesetting, and review of the resulting proof before it is published in its final citable form. Please note that during the production process errors may be discovered which could affect the content, and all legal disclaimers that apply to the journal pertain. 
The upsurge in alcohol use that often occurs during the first year of college has been convincingly linked to a number of negative psychosocial consequences and may negatively affect brain development. In this longitudinal functional magnetic resonance imaging (fMRI) pilot study, we examined changes in neural responses to alcohol cues across the first year of college in a normative sample of late adolescents. Participants $(\mathrm{N}=11)$ were scanned three times across their first year of college (summer, first semester, second semester), while completing a go/no-go task in which images of alcoholic and non-alcoholic beverages were the response cues. A state-of-theart effective connectivity mapping technique was used to capture spatiotemporal relations among brain regions of interest (ROIs) at the level of the group and the individual. Effective connections among ROIs implicated in cognitive control were greatest at the second assessment (when negative consequences of alcohol use increased), and effective connections among ROIs implicated in emotion processing were lower (and response times were slower) when participants were instructed to respond to alcohol cues compared to non-alcohol cues. These preliminary findings demonstrate the value of a prospective effective connectivity approach for understanding adolescent changes in alcohol-related neural processes.

\section{Keywords}

adolescence; alcohol; effective connectivity; longitudinal; college; drinking; fMRI

\section{Introduction}

Alcohol use increases dramatically during late adolescence (Brown et al., 2008; Schulenberg $\&$ Maggs, 2002). Rates of alcohol use are especially high among late adolescents who attend college (Schulenberg et al., 2001). Indeed, alcohol is a key part of college culture: Over $80 \%$ of college students have tried alcohol (Johnston, O’Malley, Bachman, \& Schulenberg, 2011) and over $40 \%$ have had one or more past-month episodes of heavy episodic drinking (i.e., five or more drinks on the same occasion; Substance Abuse and Mental Health Services Administration, 2011).

Escalations in alcohol consumption are particularly acute during the first year of college (Borsari, Murphy, \& Barnett, 2007; Fromme, Corbin, \& Kruse, 2008; Sher \& Rutledge, 2007). In one representative sample, over half of the students who were "weekend bingers" (heavy episodic drinking only on Fridays and Saturdays) during the summer before college became "heavy drinkers" (heavy episodic drinking on both weekdays and weekends) during their first semester (Cleveland, Lanza, Ray, Turrisi, \& Mallett, 2012). Moreover, about 20\% of first year students in the Harvard School of Public Health College Alcohol Study initiated alcohol use that year (Lindsay, 2006).

The upsurge in alcohol use that occurs in the first year of college has been convincingly linked to a number of negative psychosocial consequences, including hangovers (veisalgia), risk-taking behavior (e.g., driving while intoxicated, unprotected sexual activity), and poor academic performance (Mallett et al., 2011; Perkins, 2002; Turrisi, Mallett, Mastroleo, \& Larimer, 2006). Less clear - but of growing concern - are the potentially detrimental effects that late adolescent alcohol use may have on neurodevelopment (Guerri \& Pascual, 2010; Squeglia, Jacobus, \& Tapert, 2009). Changes in brain structure and function that typify healthy cognitive development occur throughout adolescence (Dahl, 2004; Giedd, 2008). Recent cross-sectional neuroimaging studies suggest that the heavy episodic drinking characteristic of adolescence damages the brain and is associated with cognitive deficits that may last into adulthood (Crego et al., 2010; McQueeny et al., 2009; Squeglia, Schweinsburg, Pulido, \& Tapert, 2011). 
A number of questions remain about the links between alcohol use and brain and psychological development in late adolescence and early adulthood. For example, it is unclear whether neural and cognitive changes occur only after extensive and prolonged alcohol consumption, or whether they can occur with moderate or irregular exposure (e.g., due to heavy episodic drinking). Studies have only begun to elucidate how alcohol-related decision-making is influenced by neural, cognitive, and affective processes that are still developing during adolescence, and how alcohol use, in turn, changes the brain and the psychological processes it subserves.

The associations between alcohol use and brain development throughout adolescence are dynamic and complex, so characterizing their nature across time is a key challenge. Approaches that involve examining spatiotemporal connectivity between neural regions are likely to be informative, as it is becoming increasingly clear that investigating such interregional relations are essential for developing a comprehensive understanding of information processing in the brain (Bressler \& Menon, 2010).

In this pilot study we utilized effective connectivity mapping to identify how the temporal interplay (based on the blood oxygen level-dependent, BOLD, signal) among spatially disparate brain regions changed in response to alcohol cues across the first year of college. Effective connectivity mapping has two major advantages over traditional approaches. First, compared to statistical parametric maps, which identify engaged brain regions, connectivity maps capture the dynamic relations among brain regions of interest (ROIs) during a task. Second, compared to functional connectivity maps, which identify ROIs that have contemporaneous relations in BOLD, effective connectivity maps identify ROIs that have contemporaneous and time-lagged relations in BOLD; both types of relations must be simultaneously considered in order to obtain accurate results in data simulations (Gates, Molenaar, Hillary, Ram, \& Rovine, 2010). We implemented these advantages with a stateof-the-art approach to effective connectivity mapping, the unified structural equation model (uSEM; Kim, Zhu, Chang, Bentler, \& Ernst, 2007), which combines traditional SEM (for contemporaneous relations) and vector autoregression (for lagged relations).

Many approaches for aggregating across individual connectivity maps assume that maps are similar across individuals in a group. But, emerging evidence suggests that brain processes are heterogeneous across individuals (Molenaar, 2004; Sporns, 2011); thus, traditional aggregation approaches may produce results that fail to describe any individual, or that contain spurious relations that do not exist for any individual (Gates \& Molenaar, 2012; Molenaar \& Campbell, 2009; Smith, 2012). In this case, the group model does not describe the ways in which individuals process information. In order to avoid this pitfall, we utilized Group Iterative Multiple Model Estimation (GIMME), a new approach for identifying group-level effective connectivity maps that incorporates individual-level variation.

These innovative modeling techniques were used to address our substantive question: How do the brain processes underlying responses to alcohol-related stimuli change across the first year of college? This question is important for several reasons. First, as discussed above, exposure to alcohol - and, by extension, to alcohol cues - increases substantially during the first year of college. Second, responses to affective stimuli (including alcohol cues) critically depend upon relations among multiple brain regions; the subcortical limbic response to appetitive stimuli is often modulated by the anterior cingulate cortex and other frontal cortical regions (Goldstein \& Volkow, 2002; Ochsner \& Gross, 2005). In this context, human neuroimaging work has identified brain regions that are important for responses to drug and alcohol cues: dorsolateral prefrontal cortex (DLPFC), dorsal and rostral anterior cingulate cortex (ACC), orbitofrontal cortex (OFC), and amygdala (Chase, Eickhoff, Laird, \& Hogarth, 2011; Goldstein \& Volkow, 2002; Simmonds, Pekar, \& Mostofsky, 2008; 
Wilson, Sayette, \& Fiez, 2004; Yalachkov, Kaiser, \& Naumer, 2012). Third, the tendency to attend to alcohol-related stimuli plays an important role in problematic drinking among adolescents (Field, Christiansen, Cole, \& Goudie, 2007; Houben, Nederkoorn, Wiers, \& Jansen, 2011; Murphy \& Garavan, 2011; Oei \& Morawska, 2004; Ray, Hanson, Hanson, \& Bates, 2010). In the present work, we develop a better understanding of the effects of alcohol cues on brain effective connectivity, and how these effects change across the first year of college.

\section{Method}

\subsection{Participants}

Eleven young adults, aged 18 to 19 years (6 women) were recruited from a study of parent socialization and alcohol use patterns in college students (procedures outlined in Mallett et al., 2011; Varvil-Weld, Mallett, Turrisi, \& Abar, 2012); there have been previous reports from sub-samples of the parent study (e.g., Abar, Turrisi, \& Abar, 2011; Ray, Turrisi, Abar, $\&$ Peters, 2009). Participants were recruited during the summer before their first year in college.

\subsection{Procedure}

Participants provided data in each of three sessions (waves) during their first year of college: (1) in August, before the start of classes $(\mathrm{N}=11)$; (2) in late October/early November, during the first semester $(\mathrm{N}=10)$; (3) in late January/early February, during the second semester $(\mathrm{N}=10)$. Two participants provided data for wave 1 and either wave 2 or wave 3 . The task and procedure were identical for all three waves. Participants provided written informed consent under a protocol (\#22262) approved by the Penn State Institutional Review Board.

Participants engaged in a go/no-go task while in the magnetic resonance imaging (MRI) scanner. The response cues were full color photographs from a database created by Pulido and colleagues (2010) depicting alcoholic or non-alcoholic beverages; the alcohol and nonalcohol images were matched on valence, arousal, familiarity, complexity, color, and brightness. Participants were instructed to respond (press a button on the grip device) as quickly and accurately as possible to one image type: In the respond alcohol condition, participants responded to images of alcoholic beverages; in the respond neutral condition, participants responded to images of non-alcoholic beverages. Each participant contributed data to both conditions; condition order was counter-balanced across participants. A block design was used, and the sequence of image presentation was identical in both conditions. Each condition consisted of four blocks, and each block consisted of a sequence of 40 images (60 seconds). Images were each presented for 1,000 milliseconds with 500 millisecond interstimulus intervals during which a fixation cross was presented; the fixation cross was also presented for 21 seconds between blocks. In two blocks within each condition, "go" cues were presented $75 \%$ of the time; in the other two blocks, "go" cues were presented $25 \%$ of the time. Image presentation was controlled by E-Prime 2.0 (Psychology Software Tools, Inc.).

\subsection{Measures and Data Analysis Plan}

2.3.1. Alcohol-Related Behavior-Information regarding alcohol use was collected as part of the parent study and is presented here to confirm expectations about increased drinking during the first year of college. Negative consequences of alcohol use were determined by a sum score on the Young Adult Alcohol Problems Screening Test (YAAPST; Hurlbut \& Sher, 1992). The YAAPST contains 33 items about the negative experiences occurring from alcohol use (e.g., blacking out, having sex with someone one 
would not ordinarily have sex with, receiving a lower grade on an exam than one would ordinarily receive); items were answered on a scale from 0 ("No, Never") to 9 ("40 times or more in the past year"). Data on the YAAPST were collected multiple times throughout participants' first two years of college. The first two waves of YAAPST data collection occurred close in time to the first two waves of MRI data collection: in June, before the start of classes, and in December of the first semester. The third wave of YAAPST data collection occurred in October of sophomore year; this was after the third wave of neuroimaging assessment but the data allowed for an examination of change in alcohol use after the first year of college. Internal consistency for the YAAPST was excellent for all three waves ( $\alpha=.90, .87, .88$, respectively).

Changes in negative consequences of alcohol use across waves were examined using a oneway analysis of variance (ANOVA), with wave (1-3) as the within-participant factor and the YAAPST sum score as the dependent variable. Significant results were probed with pairwise $t$-tests; Type I error was .05. Two participants had one assessment, and two additional participants had two assessments. The ANOVA was conducted on seven participants with complete data, but pairwise $t$-tests utilized all available data.

2.3.2. Task-Related Behavior-Responses on the go/no-go task were timed and categorized as hits, misses, correct rejections, or false alarms based upon whether a participant responded and whether the response was correct. Because the design was fully balanced, analyses were conducted on d': standardized hits minus standardized false alarms. Reaction time analyses focused only on data from correct responses. Reaction times and d' were entered as dependent variables in two two-way ANOVAs, with response condition (respond alcohol, respond neutral) and wave (1-3) as the within-participant factors for each analysis. Significant results were probed with pairwise $t$-tests; Type I error was .05. Two participants had two assessments. ANOVAs were conducted on nine participants with complete data, but pairwise $t$-tests utilized all available data.

2.3.3. Neuroimaging-MRI data were collected at the Penn State Social, Life, and Engineering Sciences Imaging Center using a research-dedicated 3-Tesla Siemens Trio scanner. A series of 220 functional MRI (fMRI) volumes were acquired using an echoplanar imaging pulse sequence (repetition time, TR=3,000 ms; echo time, TE $=30 \mathrm{~ms}$; flip angle $=70^{\circ} ; 74 \times 74$ matrix; 45 slices; $2.973 \times 2.973 \times 3 \mathrm{~mm}$ voxels). A high-resolution T1weighted magnetization prepared rapid acquisition gradient echo structural scan was also collected $\left(\mathrm{TR}=2300 \mathrm{~ms} ; \mathrm{TE}=3.46 \mathrm{~ms}\right.$; flip angle $=9^{\circ} ; 256 \times 224$ matrix; 160 slices; $.895 \times$. $895 \times .9 \mathrm{~mm}$ voxels) for registering each participant's functional data to standard (Montreal Neurological Institute, MNI) space.

Preprocessing of fMRI data was conducted using the fMRI Expert Analysis Tool Version 5.98, part of FSL (Oxford Centre for FMRI of the Brain, FMRIB, Software Library, www.fmrib.ox.ac.uk/fsl) before extracting BOLD time series data from ROIs for connectivity analyses. The following preprocessing steps were applied to the data: motion correction using FMRIB's Linear Image Registration Tool (Jenkinson, Bannister, Brady, \& Smith, 2002); non-brain removal using FMRIB's Brain Extraction Tool (Smith, 2002); highpass temporal filtering (performed separately for each half of the time series); spatial smoothing using a $6 \mathrm{~mm}$ full-width at half-maximum Gaussian kernel; and grand-mean intensity normalisation of the entire four-dimensional dataset by a single multiplicative factor. Registration to MNI stereotaxic space (FSL's MNI 152; T1 $2 \times 2 \times 2 \mathrm{~mm}$ ) was carried out using FMRIB's Linear Image Registration Tool (Jenkinson et al., 2002), and further refined using FMRIB's Nonlinear Image Registration Tool (Andersson, Jenkinson, \& Smith, 2007). Time series statistical analysis was carried out using FMRIB's Improved Linear Model with local autocorrelation correction (Woolrich, Ripley, Brady, \& Smith, 2001). 
Group-level analyses were conducted in order to confirm task-related brain activation. An explanatory variable (EV) was created for each response condition. EVs were not created for individual hits (go) and correct rejections (no-go) because the go/no-go task displayed two stimuli within each scanner-collected brain volume; thus, trial-level neural responses could not be reliably extracted. Each EV was convolved with a gamma function to better approximate the temporal course of the BOLD hemodynamic response, and temporal derivatives of each EV were added to the model (Miezin, Maccotta, Ollinger, Petersen, \& Buckner, 2000). Higher-level analyses were carried out using FMRIB's Local Analysis of Mixed Effects stage 1 (Woolrich, 2008). Primary contrasts of interest were response condition versus fixation (i.e., respond alcohol > rest, and respond neutral > rest) averaged across all three waves. Functional activation maps for each response condition were clustercorrected for multiple comparisons (based on Gaussian random field theory) using a threshold of $\mathrm{z}>2.3$ and a cluster significance level of $p=.05$, whole-brain corrected (Worsley, 2001). Direct comparisons of the two response conditions (respond alcohol versus respond neutral) were not conducted because the go/no-go task was not structured to allow for a clean contrast between conditions, as images of both alcoholic and non-alcoholic beverages were present in each block. Rather, the task was designed to produce a response set (i.e., identify and respond to one image category while ignoring the other) that persisted across 60 -second blocks.

Connectivity analyses were conducted using a priori ROIs extracted from MNI space (the registration to which was successful for all participants in all waves). Bilateral ROIs were selected for DLPFC $( \pm 40,18,24$; Mohanty et al., 2007), OFC ( $\pm 40,28,-14)$, and amygdala $(-24,-2,-22 ; 26,0-22)$. Midline ROIs were also selected for dorsal ACC (dACC; 4, 14, 36) and rostral ACC (rACC; -2, 44, 20; Mohanty et al., 2007). A sphere 7 voxels (14mm) in diameter was created around the center coordinate for each ROI, and the BOLD signal was averaged across this sphere for each volume. For each participant, time series data were extracted for the first (108 volumes) and second (112 volumes) halves of the experimental run, corresponding to the two response conditions. Figure 1 shows the location of the eight ROIs in MNI space.

2.3.4. Effective Connectivity-Connectivity among the eight ROIs was determined with uSEM (Kim et al., 2007), which provides a data-driven approach to hypothesis testing and can be programmed into traditional SEM software; we used LISREL (Jöreskog \& Sörbom, 1992). For a single "lag" (i.e., ROI activation at one TR predicting ROI activation at the next TR), uSEM implemented via GIMME is defined as:

$$
\eta_{i}(\mathrm{t})=\left(A_{i}+A^{g}\right) \eta_{i}+\left(\Phi_{1, i}+\Phi_{1}^{g}\right) \eta_{i}(\mathrm{t}-1)+\zeta_{i}(\mathrm{t})
$$

where $\eta_{i}(\mathrm{t})$ is the $\mathrm{p}$-variate ROI time series at time $\mathrm{t}=1,2, \ldots \mathrm{T}$, and $\mathrm{p}$ is the number of ROIs ( 8 for this study) and $\mathrm{T}$ is the length of the time series, or number of functional volumes being modeled (108 or 112 for this study); $A$ is the (p,p)-dimension matrix of contemporaneous relations among ROIs; $\Phi_{1}$ is the (p,p)-dimension matrix of lag 1 relations among ROIs; $\zeta$ is the p-variate error series assumed to contain no temporal dependencies; superscript $g$ indicates the common estimates for the group; subscript $i$ indicates the deviation from the group mean for an individual (Gates \& Molenaar, 2012; Kim et al., 2007).

Model selection at the group and individual levels was conducted with GIMME, an automated MATLAB®-based program (Mathworks, 2009b) implemented in several steps (Gates \& Molenaar, 2012). First, Lagrange Multiplier equivalents (i.e., modification indices; Sörbom, 1989) were used to identify which connections among ROIs, if freed, optimally 
improved model fits across all individuals. The probability of detecting an effect across all individuals was set at $75 \%$; selection of this criterion was informed by empirical and simulated studies on the likelihood of not identifying false positives (Hillary et al., 2011; Smith et al., 2011). The program iterated until the $75 \%$ criterion was not met. Second, the model was pruned by eliminating connections that were no longer significant for $75 \%$ of the group after other connections were freed. Third, individual-level models were estimated in a semi-confirmatory manner. All connections freed in the group model (described in the two steps above) were freed at the individual-level. The automatic search procedure within LISREL then iteratively freed connections that optimally improved model fit, according to the Lagrange Multiplier equivalents (Gates et al., 2010). Fourth, the model was pruned by eliminating individual-level connections that became nonsignificant after other individuallevel connections were freed, and a confirmatory model was fit. Final models had excellent fit to the data according to at least two of four alternative fit indices (found to be reliable in simulation studies; Brown, 2006): confirmatory fit index (CFI) $\geq .95$; non-normed fit index (NNFI) 2.95 ; standardized root mean square residual (SRMR) $\leq .05$; root mean square error of approximation (RMSEA) $\leq .10$.

Model fitting (uSEM implemented through GIMME) was conducted separately for each response condition (of two) and wave (of three); thus, six sets of final models were acquired. Connections present among ROIs in the final models were tallied in order to make inferences about neural system functioning: The frequency of connections (at the group- or individual-level, contemporaneous or lagged) among ROIs typically implicated in cognitive control (left DLPFC, right DLPFC, dACC, rACC, ) and emotion processing (left OFC, right OFC, left amygdala, right amygdala) were compared. Specifically, we counted the number of times in each of the six sets of connectivity maps that there were connections: (1) within the cognitive control network (activity in a cognitive control ROI was predicted by activity in another cognitive control ROI); (2) within the emotion processing network (activity in an emotion processing ROI was predicted by activity in another emotion processing ROI); (3) between the cognitive control and emotion processing networks (activity in a cognitive control ROI was predicted by activity in an emotion processing ROI, or vice versa). These frequencies were then entered as dependent variables in three two-way ANOVAs, with response condition (respond alcohol, respond neutral) and wave (1-3) as the withinparticipant factors for each analysis. Significant results were probed with pairwise $t$-tests; Type I error was .05. Two participants had two assessments. ANOVAs were conducted on nine participants with complete data, but pairwise $t$-tests utilized all available data.

\section{Results}

\subsection{Alcohol-Related Behavior}

Findings confirm expectations about increased negative consequences of alcohol use in the first year of college. There was significant change in YAAPST sum score across waves, $F(2,12)=6.78, p=.018, \eta_{p}{ }^{2}=.53$. Follow-up $t$-tests revealed significant increases in alcohol use from wave 1 to wave $2, t(7)=-2.65, p=.033, d=-.95$, and from wave 1 to wave $3, t(7)=-3.74, p=.007, d=-1.44$; this is shown in Figure 2 .

\subsection{Task-Related Behavior}

Correct response percentages across waves and conditions ranged from $75-100 \%(M=96 \%$, $S D=.7 \%)$. For d', there was no main effect of response condition, $F(1,8)=.93, p=.363$, no main effect of wave, $F(2,16)=.66, p=.505$, but there was an interaction between response condition and wave, $F(2,16)=4.66, p=.041, \eta_{p}{ }^{2}=.37$. Follow-up $t$-tests revealed that in the respond alcohol condition, d' increased from wave $1(M=3.62, S D=.50)$ to wave $3(M$ $=4.01, S D=.69), t(9)=-2.40, p=.040, d=-.80$. 
Response times (ms) showed a main effect of response condition, $F(1,8)=21.00, p=.002$, $\eta_{p}{ }^{2}=.72$, with slower responses during respond alcohol $(M=540, S D=37)$ than respond neutral $(M=519, S D=42)$, and a main effect of wave, $F(2,16)=5.47, p=.023, \eta_{p}^{2}=.41$, with decreases in response times from wave $1(M=540, S D=33)$ to wave $3(M=517, S D$ $=48), t(9)=2.48, p=.035, d=.87$, and from wave $2(M=534, S D=40)$ to wave $3, t(8)=$ $2.79, p=.024, d=1.00$. There was no interaction, $F(2,16)=.76, p=.763$.

\subsection{Neuroimaging}

Group-level analyses confirm task-related brain activation. Figure 1 shows cluster-corrected brain activity during the respond alcohol and respond neutral conditions (each versus fixation) averaged across all three waves. Each of the eight ROIs was overlaid on the cluster-corrected brain activity to visualize location of a priori ROIs in relation to taskrelated activation. Although some ROIs appear to be in brain regions where there is not robust group-level brain activation, effective connectivity analyses were conducted because group-level analyses often fail to detect important individual-level patterns of brain activity (Gonzalez-Castillo et al., 2012), and the aim of this pilot study was to capture interregional covariation within individuals rather than group-level effects.

\subsection{Effective Connectivity}

Final connectivity models had excellent fit. Table 1 shows the average fit indices for each set of final models. For illustrative purposes, the final model for a single participant during the respond alcohol condition at wave 1 is displayed in Figure 3. Arrows in the model represent BOLD activity in one ROI predicting BOLD activity in another ROI; they represent statistical prediction (not direction of information flow) and have corresponding beta-values. In Figure 3, there are 6 connections within the cognitive control network, 6 connections within the emotion processing network, and 9 between-network connections.

For connections within the cognitive control network across participants, there was a main effect of wave, $F(2,16)=5.69, p=.025, \eta_{p}{ }^{2}=.42$, with more connections at wave 2 than at wave $1, t(9)=-2.46, p=.036, d=-.78$, or at wave $3, t(8)=2.70, p=.027, d=.91$, no main effect of response condition, $F(1,8)=.18, p=.681$, and no interaction between response condition and wave, $F(2,16)=.18, p=.743$; this is shown in Figure 4 .

For connections within the emotion processing network, there was a main effect of response condition, $F(1,8)=7.19, p=.028, \eta_{p}^{2}=.47$, with more connections during respond neutral $(M=6.53, S D=1.15)$ than respond alcohol $(M=6.06, S D=1.02)$, no main effect of wave, $F(2,16)=1.99, p=.186$, and no interaction between response condition and wave, $F(2,16)$ $=.13, p=.846$.

For connections between the cognitive control and emotion processing networks, there was no main effect of response condition, $F(1,8)=.71, p=.425$, no main effect of wave, $F(2,16)$ $=1.37, p=.282$, and no interaction between response condition and wave, $F(2,16)=1.33, p$ $=.292$.

\section{Discussion}

In this prospective fMRI pilot study we identified changes across the first year of college in alcohol-related brain networks. In each of three waves, participants completed a go/no-go task with images of alcoholic (respond alcohol condition) and non-alcoholic (respond neutral condition) beverages as the "go" cues. At each wave and condition, we implemented a state-of-the-art effective connectivity mapping technique that captured group and individual as well as lagged and contemporaneous connections among ROIs (Gates et al., 2010; Gates \& Molenaar, 2012; Kim et al., 2007). 
Connections among ROIs were classified according to their presence within the cognitive control network (bilateral DLPFC, rACC, dACC), within the emotion processing network (bilateral OFC, bilateral amygdala), or between the cognitive control and emotion processing networks. Within the cognitive control network, there were more connections at wave 2 than at waves 1 and 3, suggesting that the first-semester transition into the college environment (wave 2) is an important period of change for alcohol-related neural processes. Waves 1 and 3 did not differ, perhaps reflecting that alcohol-related neural processes habituated to the college environment. Within the emotion processing network, there were more connections during the respond neutral condition than the respond alcohol condition across waves. Findings regarding brain connectivity in the two networks should be interpreted together: Brain regions involved in emotion processing showed less connectivity when participants responded to seemingly-dominant alcohol cues than when they responded to non-alcohol cues; but after an initial period of heightened, real-life exposure to alcohol cues (first semester of college), participants needed to heavily recruit brain regions involved in cognitive control in order to overcome the prepotent alcohol stimuli and follow "go" cue instructions.

Regarding task performance, participants improved across the study, showing faster reaction times in wave 3 than waves 1 and 2. Despite these linear practice effects, participants showed the greatest number of connections among ROIs within the cognitive control network at wave 2 , suggesting that connectivity among cognitive control ROIs was more than a simple reflection of faster task performance. Moreover, reaction times were slower during the respond alcohol than the respond neutral condition, and response accuracy improved from wave 1 to wave 3 for the respond alcohol condition. This suggests that the reduced connectivity among ROIs within the emotion processing network during the respond alcohol condition (compared to the respond neutral condition) was linked to slower task performance and increasing accuracy. But, the interpretation of this finding is tentative because task performance could not be explicitly examined in the effective connectivity models (due to the block design of the task). This is an important area for future work.

Regarding alcohol-related behavior, negative consequences of alcohol use increased from the summer before college (wave 1) to the first semester of college (wave 2), then remained relatively stable through the beginning of sophomore year (wave 3 ). This is consistent with alcohol use data reported in the parent sample (Mallett et al., 2011; Varvil-Weld et al., 2012) and other large samples (Borsari et al., 2007; Cleveland et al., 2012; Fromme et al., 2008; Lindsay, 2006; Sher \& Rutledge, 2007). This increase in negative consequences of alcohol use occurred precisely when the greatest number of connections among cognitive control brain regions was recruited to complete the alcohol-related go/no-go task.

Connections between ROIs in the cognitive control network and ROIs in the emotion processing network did not differ by response condition or wave. This is consistent with the idea of neural small-world efficiency (see Sporns, 2011): Changes might occur within brain networks in order to maintain the efficiency of communication between brain networks. But, we may have failed to see differences in between-network relations because the grouping of ROIs into cognitive control and emotion processing networks is not absolute. For example, the ACC and DLPFC are engaged during some emotion-related tasks (Compton et al., 2003; Davidson, 2004). Results might also differ if other ROIs were included in the cognitive control (e.g., inferior frontal gyrus) and emotion processing (e.g., ventromedial prefrontal cortex) networks. Given the pilot nature of this study, we included a small and equal number of ROIs in each network, and it will be important for future work to expand upon this.

We suggested that greater exposure to alcohol cues early in college changes effective connectivity among brain regions underlying the response to those cues, but developmental 
changes in brain activity may also influence the perception of alcohol cues. The narrow age range of this sample and the longitudinal design of this study, however, suggest that this is not the case. Further work in this area could disentangle contextual and developmental influences on alcohol-related brain activity by comparing adolescents who do (versus those who do not) go to college, examining brain-behavior links in large samples across long periods of time (e.g., annual assessment through high school and college), or collecting several waves of data in a short time period (e.g., monthly assessment through the summer and first semester of college).

This pilot study demonstrates the feasibility and value of examining prospective changes in the effective connectivity of alcohol-related networks across the first year of college. Future work can expand upon this approach in several ways. First, this approach can be generalized to other tasks, brain networks, ROIs, and samples. Second, event-related fMRI data can be modeled within an effective connectivity framework in order to capture neural activity associated with specific, time-locked task responses (Gates, Molenaar, Hillary, \& Slobounov, 2011). Third, participant behaviors (e.g., alcohol use) can be related to connections within individual-level connectivity maps. Longitudinal mapping of individuallevel brain connectivity has the potential to increase our understanding of the dynamic brain processes that underlie adolescent alcohol-related behavior.

\section{Acknowledgments}

We thank the following people who contributed to this work: Susan Tapert, Caitlin Abar, and Susan Lemieux collaborated on study design; Jeremy Fesi ran participants; Lindsey Varvil-Weld, Nichole Scaglione, and Aimee Read helped with recruitment and data management; Amanda Thomas helped with data management. We also thank the participants.

\section{Role of Funding Sources}

Funding for this research was provided by NIAAA grant R01AA015737 to Rob Turrisi, instrumentation funded by the National Science Foundation through grant OCI-0821527, Penn State Institute of the Neurosciences, Penn State Social Science Research Institute, and Penn State Social, Life, and Engineering Sciences Imaging Center 3-Tesla

Magnetic Resonance Imaging Facility. Funding sources did not play a role in study design, data collection, analysis, or interpretation, or in the writing or decision to submit this article for publication.

\section{References}

Abar C, Turrisi R, Abar B. Brief report: Tailgating as a unique context for parental modeling on college student alcohol use. Journal of Adolescence. 2011; 34(5):1103-1106. [PubMed: 20561673]

Andersson, JLR.; Jenkinson, M.; Smith, SM. Nonlinear registration, aka spatial normalisation. 2007. FMRIB technical report TR07JA2 from www.fmrib.ox.ac.uk/analysis/techrep

Borsari B, Murphy JG, Barnett NP. Predictors of alcohol use during the first year of college: Implications for prevention. Addictive Behaviors. 2007; 32(10):2062-2086. [PubMed: 17321059]

Bressler SL, Menon V. Large-scale brain networks in cognition: Emerging methods and principles. Trends in Cognitive Sciences. 2010; 14(6):277-290. [PubMed: 20493761]

Brown SA, McGue M, Maggs J, Schulenberg J, Hingson R, Swartzwelder S, et al. A developmental perspective on alcohol and youths 16 to 20 years of age. Pediatrics. 2008; 121(4):S290-310. [PubMed: 18381495]

Brown, TA. Confirmatory factor analysis for applied research. New York: Guilford Press; 2006.

Chase HW, Eickhoff SB, Laird AR, Hogarth L. The neural basis of drug stimulus processing and craving: An activation likelihood estimation meta-analysis. Biological Psychiatry. 2011; 70(8):785793. [PubMed: 21757184]

Cleveland MJ, Lanza ST, Ray AE, Turrisi R, Mallett KA. Transitions in first-year college student drinking behaviors: Does pre-college drinking moderate the effects of parent- and peer-based intervention components? Psychology of Addictive Behaviors. 2012; 26(3):440-450. [PubMed: 22061340] 
Compton RJ, Banich MT, Mohanty A, Milham MP, Herrington J, Miller GA, et al. Paying attention to emotion: An fMRI investigation of cognitive and emotional stroop tasks. Cognitive, Affective, and Behavioral Neuroscience. 2003; 3(2):81-96.

Crego A, Rodriguez-Holguin S, Parada M, Mota N, Corral M, Cadaveira F. Reduced anterior prefrontal cortex activation in young binge drinkers during a visual working memory task. Drug and Alcohol Dependence. 2010; 109(1-3):45-56. [PubMed: 20079980]

Dahl RE. Adolescent brain development: A period of vulnerabilities and opportunities. Keynote address. Annals of the New York Academy of Sciences. 2004; 1021:1-22. [PubMed: 15251869]

Davidson RJ. What does the prefrontal cortex "do" in affect: Perspectives on frontal EEG asymmetry research. Biological Psychology. 2004; 67(1-2):219-233. [PubMed: 15130532]

Field M, Christiansen P, Cole J, Goudie A. Delay discounting and the alcohol stroop in heavy drinking adolescents. Addiction. 2007; 102(4):579-586. [PubMed: 17309540]

Fromme K, Corbin WR, Kruse MI. Behavioral risks during the transition from high school to college. Developmental Psychology. 2008; 44(5):1497-1504. [PubMed: 18793080]

Gates KM, Molenaar PC, Hillary FG, Ram N, Rovine MJ. Automatic search for fMRI connectivity mapping: An alternative to Granger causality testing using formal equivalences among SEM path modeling, VAR, and unified SEM. NeuroImage. 2010; 50(3):1118-1125. [PubMed: 20060050]

Gates KM, Molenaar PCM. Group search algorithm recovers effective connectivity maps for individuals in homogeneous and heterogeneous samples. NeuroImage. 2012; 63(1):310-319. [PubMed: 22732562]

Gates KM, Molenaar PCM, Hillary FG, Slobounov S. Extended unified SEM approach for modeling event-related fMRI data. NeuroImage. 2011; 54(2):1151-1158. [PubMed: 20804852]

Giedd JN. The teen brain: Insights from neuroimaging. Journal of Adolescent Health. 2008; 42(4): 335-343. [PubMed: 18346658]

Goldstein RZ, Volkow ND. Drug addiction and its underlying neurobiological basis: Neuroimaging evidence for the involvement of the frontal cortex. American Journal of Psychiatry. 2002; 159(10): 1642-1652. [PubMed: 12359667]

Gonzalez-Castillo J, Saad ZS, Handwerker DA, Inati SJ, Brenowitz N, Bandettini PA. Whole-brain, time-locked activation with simple tasks revealed using massive averaging and model-free analysis. Proceedings of the National Academy of Sciences of the United States of America. 2012; 109(14):5487-5492. [PubMed: 22431587]

Guerri C, Pascual M. Mechanisms involved in the neurotoxic, cognitive, and neurobehavioral effects of alcohol consumption during adolescence. Alcohol. 2010; 44(1):15-26. [PubMed: 20113871]

Hillary FG, Medaglia JD, Gates K, Molenaar PC, Slocomb J, Peechatka A, et al. Examining working memory task acquisition in a disrupted neural network. Brain. 2011; 134:1555-1570. [PubMed: 21571783]

Houben K, Nederkoorn C, Wiers RW, Jansen A. Resisting temptation: Decreasing alcohol-related affect and drinking behavior by training response inhibition. Drug and Alcohol Dependence. 2011; 116(1-3):132-136. [PubMed: 21288663]

Hurlbut SC, Sher KJ. Assessing alcohol problems in college students. Journal of American College Health. 1992; 41(2):49-58. [PubMed: 1460173]

Jenkinson M, Bannister P, Brady M, Smith S. Improved optimization for the robust and accurate linear registration and motion correction of brain images. NeuroImage. 2002; 17(2):825-841. [PubMed: 12377157]

Johnston, LD.; O'Malley, PM.; Bachman, JG.; Schulenberg, JE. Monitoring the Future national survey results on drug use, 1975-2010. College students and adults ages 19-50. Vol. II. Ann Arbor: Institute for Social Research, The University of Michigan; 2011.

Jöreskog, KG.; Sörbom, D. LISREL. Scientific Software International, Inc; 1992.

Kim J, Zhu W, Chang L, Bentler PM, Ernst T. Unified structural equation modeling approach for the analysis of multisubject, multivariate functional MRI data. Human Brain Mapping. 2007; 28(2): 85-93. [PubMed: 16718669]

Lindsay V. Factors that predict freshmen college students' preference to drink alcohol. Journal of Alcohol and Drug Education. 2006; 50(4):7-19. 
Mallett KA, Marzell M, Varvil-Weld L, Turrisi R, Guttman K, Abar C. One-time or repeat offenders? An examination of the patterns of alcohol-related consequences experienced by college students across the freshman year. Addictive Behaviors. 2011; 36(5):508-511. [PubMed: 21276662]

Mathworks. Matlab. The Mathworks, Inc; 2009b.

McQueeny T, Schweinsburg BC, Schweinsburg AD, Jacobus J, Bava S, Frank LR, et al. Altered white matter integrity in adolescent binge drinkers. Alcoholism, Clinical and Experimental Research. 2009; 33(7):1278-1285.

Miezin FM, Maccotta L, Ollinger JM, Petersen SE, Buckner RL. Characterizing the hemodynamic response: Effects of presentation rate, sampling procedure, and the possibility of ordering brain activity based on relative timing. NeuroImage. 2000; 11(6):735-759. [PubMed: 10860799]

Mohanty A, Engels AS, Herrington JD, Heller W, Ho MH, Banich MT, et al. Differential engagement of anterior cingulate cortex subdivisions for cognitive and emotional function. Psychophysiology. 2007; 44(3):343-351. [PubMed: 17433093]

Molenaar PCM. A manifesto on psychology as idiographic science: Bringing the person back into scientific psychology, this time forever. Measurement. 2004; 2(4):201-218.

Molenaar PCM, Campbell CG. The new person-specific paradigm in psychology. Current Directions in Psychological Science. 2009; 18(2):112-117.

Murphy P, Garavan H. Cognitive predictors of problem drinking and audit scores among college students. Drug and Alcohol Dependence. 2011; 115(1-2):94-100. [PubMed: 21145183]

Ochsner KN, Gross JJ. The cognitive control of emotion. Trends in Cognitive Sciences. 2005; 9(5): 242-249. [PubMed: 15866151]

Oei TP, Morawska A. A cognitive model of binge drinking: The influence of alcohol expectancies and drinking refusal self-efficacy. Addictive Behaviors. 2004; 29(1):159-179. [PubMed: 14667427]

Perkins HW. Surveying the damage: A review of research on consequences of alcohol misuse in college populations. Journal of Studies on Alcohol. 2002; (Supplement 14):91-100. [PubMed: 11925064]

Pulido C, Brown SA, Cummins K, Paulus MP, Tapert SF. Alcohol cue reactivity task development. Addictive Behaviors. 2010; 35(2):84-90. [PubMed: 19800172]

Ray AE, Turrisi R, Abar B, Peters KE. Social-cognitive correlates of protective drinking behaviors and alcohol-related consequences in college students. Addictive Behaviors. 2009; 34(11):911-917. [PubMed: 19540676]

Ray S, Hanson C, Hanson SJ, Bates ME. FMRI BOLD response in high-risk college students (part 1): During exposure to alcohol, marijuana, polydrug and emotional picture cues. Alcohol and Alcoholism. 2010; 45(5):437-443. [PubMed: 20729530]

Schulenberg JE, Maggs JL. A developmental perspective on alcohol use and heavy drinking during adolescence and the transition to young adulthood. Journal of Studies on Alcohol. 2002; (Supplement 14):54-70.

Schulenberg JE, Maggs JL, Long SW, Sher KJ, Gotham HJ, Baer JS, et al. The problem of college drinking: Insights from a developmental perspective. Alcoholism, Clinical and Experimental Research. 2001; 25(3):473-477.

Sher KJ, Rutledge PC. Heavy drinking across the transition to college: Predicting first-semester heavy drinking from precollege variables. Addictive Behaviors. 2007; 32(4):819-835. [PubMed: 16860940]

Simmonds DJ, Pekar JJ, Mostofsky SH. Meta-analysis of go/no-go tasks demonstrating that fMRI activation associated with response inhibition is task-dependent. Neuropsychologia. 2008; 46(1): 224-232. [PubMed: 17850833]

Smith SM. Fast robust automated brain extraction. Human Brain Mapping. 2002; 17(3):143-155. [PubMed: 12391568]

Smith SM. The future of fMRI connectivity. NeuroImage. 2012; 62(2):1257-1266. [PubMed: 22248579]

Smith SM, Miller KL, Salimi-Khorshidi G, Webster M, Beckmann CF, Nichols TE, et al. Network modelling methods for fMRI. NeuroImage. 2011; 54(2):875-891. [PubMed: 20817103]

Sörbom D. Model modificiation. Psychometrika. 1989; 54(3):371-384. 
Sporns, O. Networks of the brain. Cambridge, MA: MIT Press; 2011.

Squeglia LM, Jacobus J, Tapert SF. The influence of substance use on adolescent brain development. Clinical EEG and Neuroscience. 2009; 40(1):31-38. [PubMed: 19278130]

Squeglia LM, Schweinsburg AD, Pulido C, Tapert SF. Adolescent binge drinking linked to abnormal spatial working memory brain activation: Differential gender effects. Alcoholism, Clinical and Experimental Research. 2011; 35(10):1831-1841.

Substance Abuse and Mental Health Services Administration. Results from the 2010 National Survey on Drug Use andHhealth: Summary of national findings. Rockville, MD: Substance Abuse and Mental Health Services Administration; 2011.

Turrisi R, Mallett KA, Mastroleo NR, Larimer ME. Heavy drinking in college students: Who is at risk and what is being done about it? Journal of General Psychology. 2006; 133(4):401-420. [PubMed: 17128959]

Varvil-Weld L, Mallett KA, Turrisi R, Abar CC. Using parental profiles to predict membership in a subset of college students experiencing excessive alcohol consequences: Findings from a longitudinal study. Journal of Studies on Alcohol and Drugs. 2012; 73(3):434-443. [PubMed: 22456248]

Wilson SJ, Sayette MA, Fiez JA. Prefrontal responses to drug cues: A neurocognitive analysis. Nature Neuroscience. 2004; 7(3):211-214.

Woolrich M. Robust group analysis using outlier inference. NeuroImage. 2008; 41(2):286-301. [PubMed: 18407525]

Woolrich MW, Ripley BD, Brady M, Smith SM. Temporal autocorrelation in univariate linear modeling of fMRI data. NeuroImage. 2001; 14(6):1370-1386. [PubMed: 11707093]

Worsley, KJ. Statistical analysis of activation images. In: Jezzard, P.; Matthews, PM.; Smith, SM., editors. Functional MRI: An introduction to methods. New York, NY: Oxford University Press; 2001.

Yalachkov Y, Kaiser J, Naumer MJ. Functional neuroimaging studies in addiction: Multisensory drug stimuli and neural cue reactivity. Neuroscience and Biobehavioral Reviews. 2012; 36(2):825-835. [PubMed: 22198678] 


\section{Research Highlights}

- Increased alcohol use during college negatively affects the adolescent brain

- First year students were scanned three times, while completing a go/no-go task

- Connectivity within the emotion network depended on response cue (alcohol, neutral)

- Connectivity within the cognitive control network changed across the first year

- Longitudinal connectivity mapping provides insight into alcohol-related behavior 

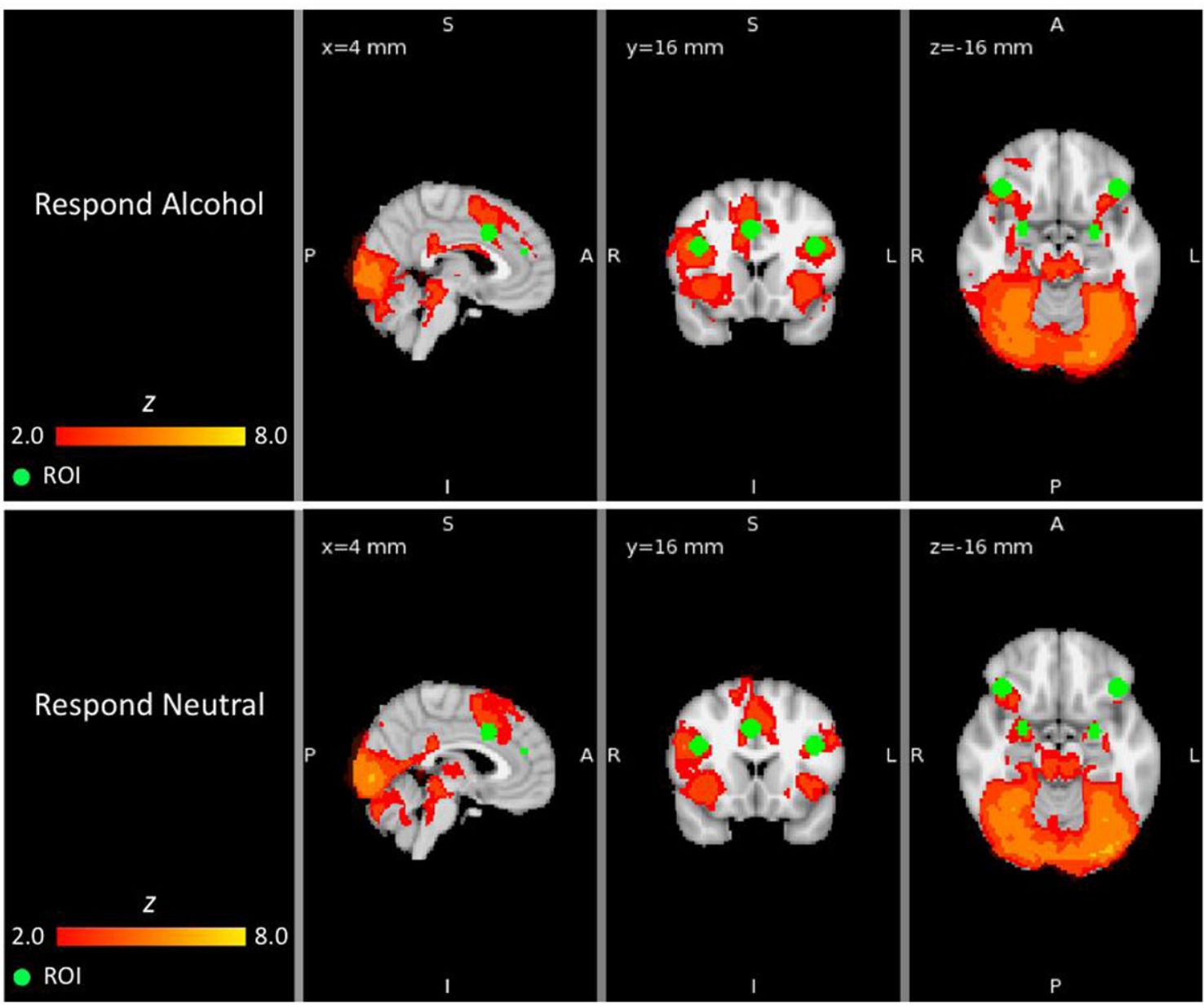

Figure 1.

ROIs and cluster-corrected brain activity (versus fixation) averaged across three waves during the respond alcohol (top) and respond neutral (bottom) task conditions overlaid on an MNI template brain. ROIs are the same in both images, with dACC and RACC in the sagittal slice (left), left and right DLPFC and dACC in the coronal slice (middle), and left and right $\mathrm{OFC}$ and left and right amygdala in the axial slice (right). 


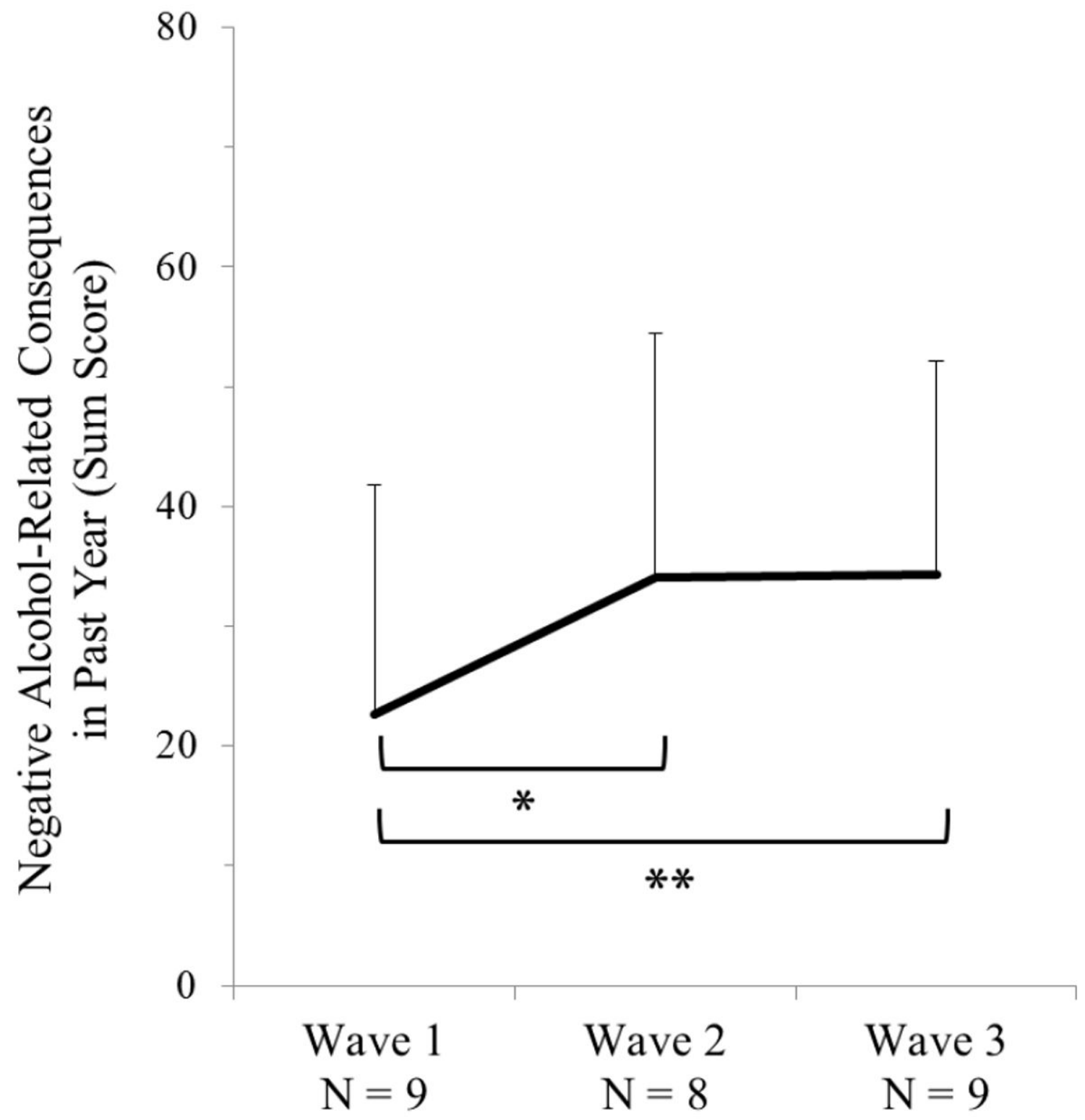

Figure 2.

Effect of wave on mean negative consequences of alcohol use in the past year. Bars represent standard deviations. Differences significant by paired $t$-test: $* p<.05 ; * * p<.01$. 


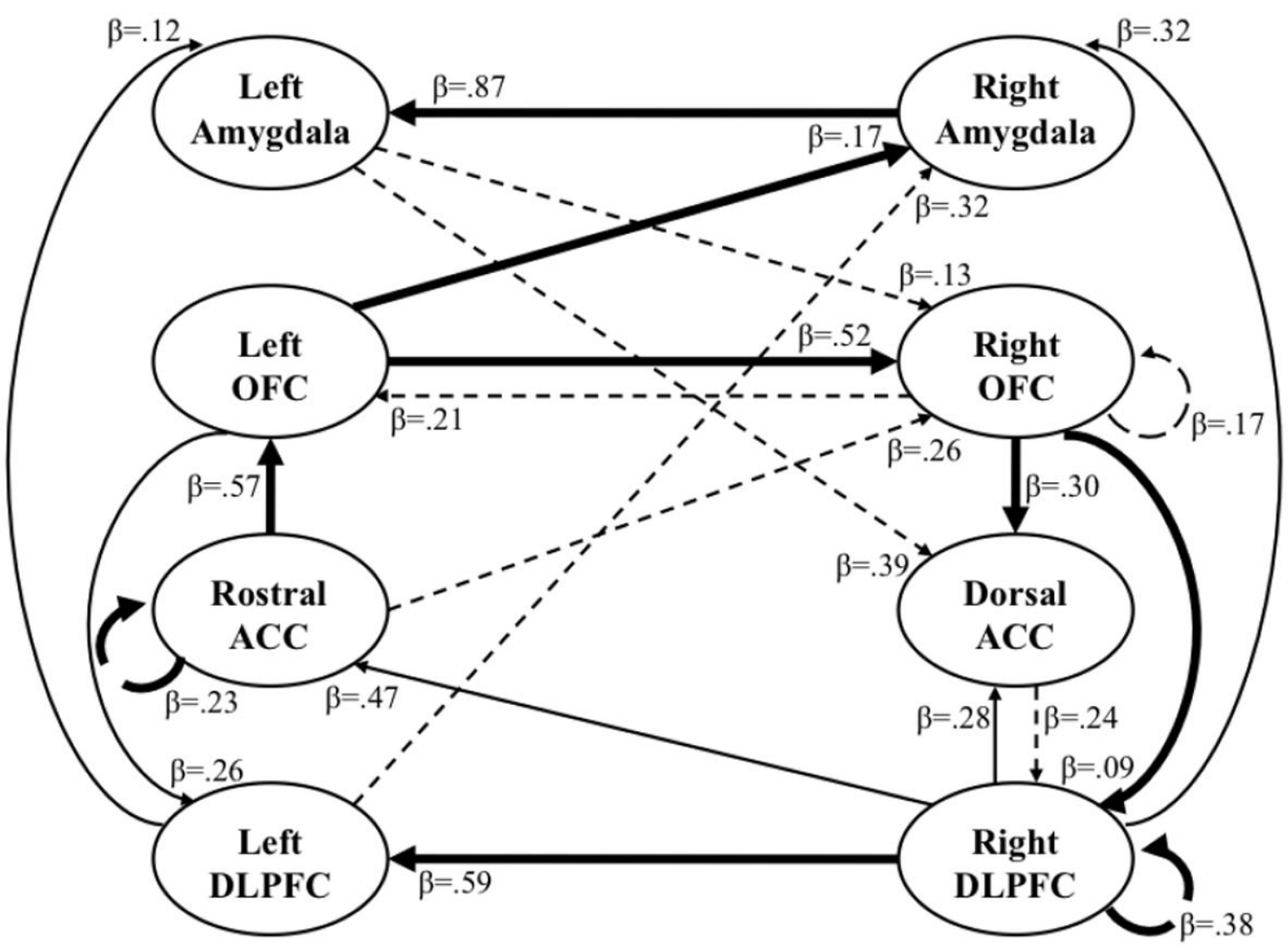

Figure 3.

A final effective connectivity map for the respond alcohol condition at wave 1 for a single participant. The model fit the data well: $\mathrm{CFI}=1.00, \mathrm{NNFI}=1.00, \mathrm{SRMR}=.050, \mathrm{RMSEA}=$. 000. Thick arrows represent group-level connections; thin arrows represent individual-level connections; solid lines represent contemporaneous relations; dashed lines represent lagged relations; all arrows have beta-values associated with them. 


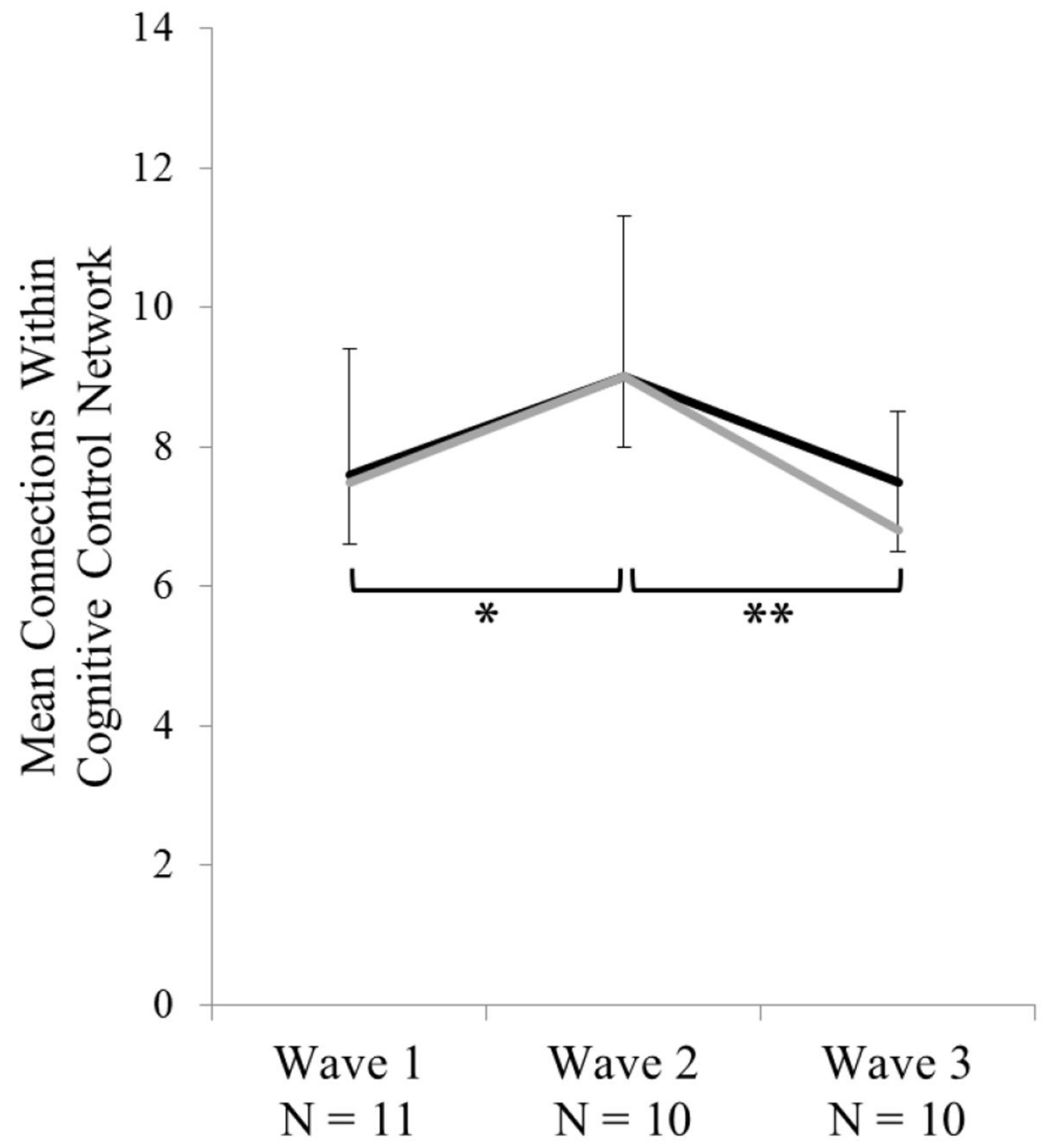

Figure 4.

Effects of response condition and wave on mean connections among ROIs within the cognitive control network (bilateral DLPFC, rACC, dACC). Black lines represent connections present during the respond alcohol condition, with negative standard deviation bars; gray lines represent connections present during the respond neutral condition, with positive standard deviation bars. Differences significant by paired $t$-test: $* p<.05 ; * * p<.01$. 
